most active, and which were exposed to irritation and pressure that most frequently suffered from perichondritis, whether primary or secondary. Further, it was in elderly people, when there was more or less tendency to ossification, that perichondritis was usually seen. It had been alleged that intralaryngeal manipulations were not an infrequent cause of perichondritis; in the speaker's opinion such cases must be very exceptional, and for his part he had never seen one or seen one recorded.Mr. LENNOX Brown was of opinion that cases of primary perichondritis was exceedingly rare; and he could only recall one case of the simple nature, of which he felt no doubt, namely, that of a boy who was attacked after going to sleep when in a state of perspiration in a hay field, exposed to an east wind. With regard to diagnosis, he did not attach so much importance as Dr. Hall to inter-arytenoid infiltration as distinguishing tuberculous perichondritis from syphilitic, because that situation was a very favourable one for syphilitic gummata. There was, however, as a rule, far more hyperæmia in syphilitic cases than in the tuberculous. A very early symptom, and one of great diagnostic importance, was subglottic swelling of the vocal cord of the effected side. Difficulty and pain in swallowing, without presence of new growth or of ulceration (malignant), or of swollen epiglottis (tubercular), were of high diagnostic importance. He thought that abscess in the case of cancer of the larynx was rare, and also that enlarged cervical glands but very rarely suppurated in this disease. He doubted over-exertion of the voice as a cause of laryngeal perichondritis, and believed that unilateral inflammation of one vocal cord, especially when recurrent, was almost invariably due to syphilis. He would not admit elimination of a syphilitic dyscrasia by negative results obtained from administration of iodide of potassium alone, but only after a steady course of mercury, either internally or by inunction, had been pursued without effect. In his experience the cartilage most frequently affected was the cricoid in its posterior aspect; next the arytenoid and cricoarytenoid articulations. Doubtless Dr. Spicer's remarks as to irritation of food, predisposed in some cases to the preference of this situation for benign perichondrial inflammations, were correct; the same, indeed, obtained in malignant disease.-The PrEsident said he thought a great distinction should be made between two conditions of perichondritis which Dr. Hall had classed together, namely, that which occurred in the course of the advance of cancer, and which depended on the extension of the primary disease to or through the perichondrium; and the perichondritis which occurred in association with, or as a sequel to, typhoid and other fevers. In this form of perichondritis there was not usually any previous affection of the larynx, and the inflammation appeared, so to speak, to fall bodily on the cartilage or its covering as tissues specially liable to be affected by the poison of the primary disease. This was the more curious when it was taken into account how very rare a disease perichondritis was in the human body. There was quite a large area of perichondrium covering the tarsal cartilages, the pinna of the ear, the nasal and laryngeal cartilages, and the cartilages of the ribs. But inflammation of the perichondrium covering any of these structures, unless it were that covering the cartilages of the larynx, was scarcely ever met with. Yet several of the parts covered with perichondrium were much exposed to cold and injury. The rarity of perichondritis was the more singular when it was taken into account how frequent inflammation of the periosteum was in certain situations. Taking all these circumstances into consideration, he quite agreed with Mr. Browne that every case of simple primary perichondritis of the larynx should be regarded with suspicion, and should only be admitted in the absence of every predisposing cause such as syphilis, fever, and the like.

\section{THYROTOMY IN THE TREATMENT OF}

\section{LARYNGEAL CANCER.}

Read in the Section of Laryngology at the Annual Meeting of the British Medical Association held in Leeds, August, 1889. By SIR WILLIAM STOKES, M.D.,

Professor of Surgery at the Royal College of Surgeons, Ireland, etc.

OF the surgical questions which await time and experience for their satisfactory settlement, the one in reference to the merits of thyrotomy in the treatment of laryngeal cancer stands in a foremost place. In connection with this topic two distinct and hos- tile camps are occupied by authorities, some advocating exclusively an endo-laryngeal method for the removal of malignant laryngeal neoplasms, while others stoutly maintain that such a procedure is only a " sort of artistic possibility of little practical value." Between these two extremes of opinion the principles that should guide us and the practice we should adopt in such cases will doubtless be established and the mists which contribute so largely to maintain the existing unfortunate chaos of surgical opinion on the question ultimately be satisfactorily cleared away. In cases of malignant growth thyrotomy, Dr. F. Semon has observed, "should not be attemptea," and this very decided opinion is practically endorsed by Gottstein, who has stated that "extirpation of malignant tumours by means of thyrotomy has not, judging by the results so far obtained, been satisfactory. In twenty cases collected by Bruns, in two death occurred soon after the operation; in one case only was there no local recurrence, but in this case death occurred twenty-two months after from cancer affecting the suprarenal capsules and the left kidney. In the remaining eighteen cases local recurrence took place, in some after two or three weeks, and only in one was there an interval of a year and a half. Recovery of voice occurred in no instance."

Sir Morell Mackenzie has published a very discouraging statistical table of cases of thyrotomy. It consists of twenty-two cases of different operators; in two only of these was there a "cure" obtained, that is, there was no evidence of recurrence after three years in one, and two years and nine months in the other. Both of these cases occurred in Professor Billroth's practice. In eight others in this table, however, recurrence of the disease and death did not occur for many months after the operation, so that in nearly 50 per cent. of the entire number of these cases the results cannot be said to be altogether unsatisfactory. Mr. A. Durham has published ${ }^{2}$ an interesting and encouraging record of five successful cases in which he performed thyrotomy for the removal of intra-laryngeal growths. All the patients were under 9 years of age. These five successful cases taken with thirty-two others collected by Mr. Durham in which thyrotomy was performed give the following results, which must be regarded with much interest. In eighteen the results were quite successful, in nine partially successful, in four temporary benefit was obtained, and in the remaining six the results were uncertain or unsuccessful. This statistical result, whatever the ultimate judgment of surgeons may be as regards the operation, proves unquestionably that many of the alleged dangers of the operation are purely imaginary, and that in truth, with due precautions being taken, it is not more hazardous, or as much so, as many other accepted and frequently performed surgical operations.

As every such case is, irrespective of the result obtained, of great importance in determining the question as to the merits of the operation, more particularly in connection with malignant and cancerous disease, I deem the following case especially worthy of record, as it will, I think, largely aid in determining surgical opinion on the subject.

S. J., aged 50 , by occupation a butler, was admitted into the Meath Hospital, under my care, on April 25th, 1889, having been recommended to me by Dr. J. Murphy, of Dublin. The patient was suffering from aphonia, great dyspnœa, and dysphagia, any solid food being especially liable to give rise to extreme laryngeal irritation. The patient was a tall, sallow-complexioned, dark-haired individual, and had always led a steady, temperate life. He never had syphilis, and there was no evidence of there ever having been any hereditary cancerous diathesis. The patient was in a condition of extreme marasmus, owing doubtless to insuffcient nutrition during the previous six months. He attributed the trouble to a severe cold with " sore throat," after which he became aphonic. He suffered no pain in his throat, nor did efforts to talk give rise to any. Attempts at deglutition of either solid or fluid food was accompanied with much distress, owing to some of it invariably passing into the air-passages, which always gave rise to violent spasmodic cough. There was no evidence of pulmonary, cardiac, or renal disease. There was no albumen, phosphatic or lithic acid deposit in the urine. The latter was in every respect in a satisfactory condition. On examination of the throat, the larynx externally appeared abnormally prominent. There was no involvement of the submaxillary or sublingual glands but at the edge of the sterno-cleido mastoid posterior, close to the os hyoides, there was one small enlarged gland. The laryngoscopic

1 Diseases of Larynx, by Gottstein, translated by P. McBride, p. 143. 2 Med. Chir. Trans., vol. lv. 
examinations, made by my colleague, Dr. P. C. Smyly, revealed a large lobulated apparently vascular growth, situated mainly on the right side of the larynx, above and completely concealing the vocal cords. The extent of the attachment of the growth could not be accurately estimated, nor could it be ascertained if the cartilages were involved in the disease; but it was observed that, having regard to the freedom with which the tumour moved up and down during the act of respiration, the probabilities were that there was no involvement of the cartilages, and that the growth was probably not sessile but pedunculated, and could be excised without removing any of the cartilages. The condition of things found at the time of the operation, which was subsequently performed by me, verified in a remarkable degree the accuracy of the diagnosis, in making which I derived so much assistance from Dr. Smyly. The structures on the left side of the larynx were found perfectly healthy.

The appearance of the growth as seen by the laryngoscope is faithfully represented in the accompanying sketch made by Dr. Smyly.

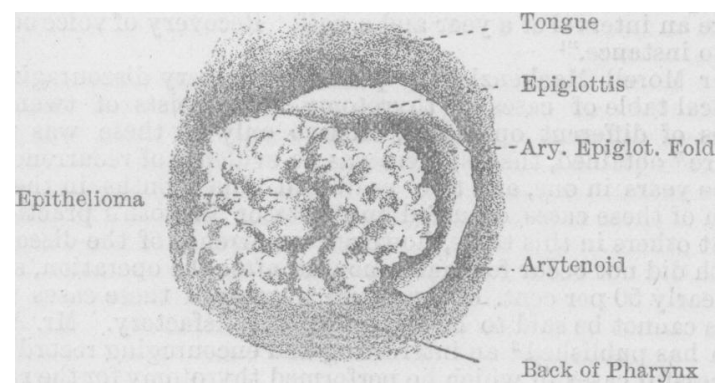

On May 10th, the dyspnœa having. distinctly increased during the two days previously, and the patient's condition from this cause as well as from the dysphagia being very critical, I performed a preliminary tracheotomy about three-quarters of an inch below the cricoid cartilage, and employed for the operation a Worthington's scalpel and grooved steel director, which I found signally facilitated the speedy introduction of a cannula, and in a fortnight subsequently, when the patient had become quite accustomed to the presence of the instrument, I substituted for it a Trendelenburg's apparatus for the twofold purpose of preventing any blood entering the air passages during the performance of thyrotomy, and through which the inhalation of an anæsthetic might also be effected. Some irritation was at first induced by Trendelenburg's tube, but this soon subsided. The patient was then brought into the operating theatre and chloroform administered. No sickness or any other trouble connected with the anæsthetic interfered with the performance of the operation.

I commenced by making an incision accurately in the mesial line, commencing at the os hyoides and carrying it down to half an inch below the cricoid cartilage. All bleeding points having been carefully secured, I opened the crico-thyroid membranes, intending to divide the thyroid alæ from below upwards with a strong pair of straight scissors. Owing, however, to the cartilages being ossified, this was not found feasible, and to effect the divigion it was necessary to employ a small-sized straight bone forceps. In this way an opening extending from the thyrohyoid space down to the cricoid cartilage, and involving the latter, was made. The thyroid alæ were now separated with blunt hook retractors and held aside.

A good view was now obtained into the interior of the larynx, and I observed with much satisfaction that the disease had not involved either of the true vocal cords. The new growth lay well above them, being attached by a pedicle to the right ala of the thyroid cartilage nearer its upper than its lower margin. On passing my right index finger into the larynx, to satisfy myself as to the size and nature of the attachment of the growth, I found that there were three of them-one apparently fully the size of a walnut and the other two about the size of kidney beans. The larger tumour was attached by a somewhat narrow pedicle, and I anticipated, therefore, little difficulty in its removal. Accordingly, with a pair of strong curved scissors I commenced to detach the pedicle.

This I did, and then passed my finger into the larynx, hoping to be able to hook it out, as it were, through the opening I had made. I did not, however, succeed in this-a circumstance which caused me some anxiety, especially as at this juncture there was very smart hæmorrhage coming from the situation where the attachment of the tumour had been severed. I then passed a curved forceps into the larynx, hoping that with it I might be able to seize and remove the detached growth. This expedient did not succeed either, and I was beginning to be apprehensive as to a possible failure attending the removal of the growth, fearing that possibly it had passed upwards into the mouth and been then swallowed by the patient. My apprehensions, however, were set at rest by my colleague, Mr. Hepburn, informing me that, while I was selecting another forceps for the removal of the tumour, it was discharged from the mouth during an effort to get rid of a quantity of mixed blood, saliva, and mucus.

The two smaller growths were situated on the posterior wall of the larynx, and these differed from the first growth removed, not only as regards size but also from being sessile. They were detached by means of a Volkmann's scoop. On their removal there was a good deal of hæmorrhage, which, however, was checked mainly by sponge pressure. Two or three small vessels at the edge of the wound were ligatured and the divided larynx brought together by six points of interrupted carbolised silk suture. The edges of the skin wound were likewise held in apposition by similar sutures, but of finer silk. Sal alembroth dressings were then applied and the patient removed to his ward.

It is unnecessary to enter into details as regards the progress of the patient after the operation. The dysphacia at once disappeared, and throughout his convalescence his condition remained perfectly afebrile and aseptic. The tracheotomy tube was removed on the tenth day after the operation, and on the sixteenth day he returned to his work. Having regard to the extent of the disease which was present, and the suspicious enlargement at the side of his neck, the probabilities certainly are that sooner or later there will be a recurrence of the disease; but still, when one considers the extremely critical condition the patient was in at the time of the operation, the result as materially prolonging his life must be looked upon as eminently satisfactory.

It has been stated that, when our manipulative dexterity in performing intra-laryngeal operations is in advance of what it is at present, thyrotomy is likely to be altogether superseded. This may possibly prove true as regards benign growths; but it is hard to conceive how it could hold good as regards tumours of a malignant type. In the removal of these in other situations, there is undoubtedly a consensus of opinion among surgeons that to augment the chances of future immunity from recurrence, a very thorough and complete removal of the disease and its neighbouring tissue is indispensable, and no exception to this established fact can exist in the case of malignant laryngeal growths. It is hard, in truth, to understand how they can be so efficiently and radically treated as is necessary, by any form of snare, forceps, or cautery, no matter how great the dexterity of the operator is, or how ingeniously devised the appliances he uses may be. What I think we are more justified in anticipating, is the knowledge of how to recognise accurately the disease while it is limited and localised, and still further by more perfect antisepticism to diminish the risks which are ever present when such operations as laryngo-fissure, partial and complete excision of the larynx, are performed.

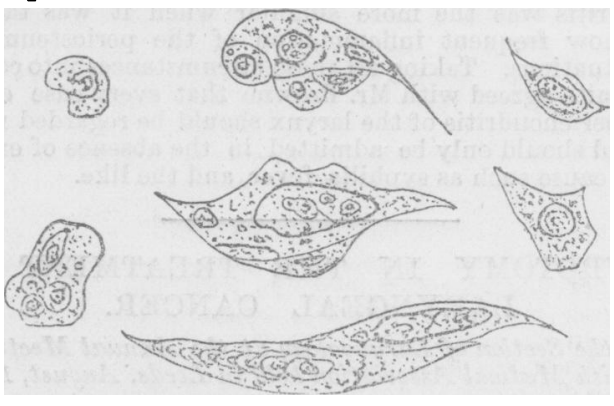

Cells from Epithelioma of Larynx.

The facts $I$ have briefly detailed in the above case, taken with the results obtained by Billroth, Durham, Von Bergmann, Rauchfuss, Köhler, Schuchardt, and others, must go far to dissipate among surgeons the feelings of hostility to the operation to which such an impulse has been given by the antagonistic opinions of many eminent laryngologists. 
In the histological examination of the three tumours 1 am much indebted for assistance to Dr. White, Pathologist to the Meath Hospital, and Dr. McKee, Curator of the Museum of the Royal College of Surgeons. Dr. White kindly furnished me with the accompanying drawing, illustrating the appearance of the cells as seen by the microscope.

Dr. McKee considers that structurally the tumours would be best described as an unisual combination of epithelioma with sarcoma. He observes: "One of the small tumours showed a growth of the superficial epithelium downwards, together with clusters of indistinctly laminated cells, having much the same characters as the superficial epithelium. The stroma, however, greatly preponderated over the cell clusters and consisted not of the usual round-celled infiltration with perhaps spindle cells and connective tissue, but of these together with epitheloid and giant cells, which bore no resemblance to the superficial epithelium. Examination of the other small tumours showed the superficial epithelium infiltrated with round cells right up the stratum corneum No ingrowth of the interpapillary processes could be detected, and although alveoli, containing epitheloid cells, were present the alveolation was indistinct as compared with the preceding specimen, and the contained cells differed totally from those of the superficial epithelium. The whole of the cells of this small tumour appeared to be of connective tissue origin, and, except for the presence of an unusually-marked alveolar arrangement in places, I should not have hesitated to call it a sarcoma. The third piece examined was taken from the large tumour at its junction with the healthy mucuous membrane. Here the superficial epithelium was perfectly normal, and the tumour was distinctly observed to have grown from below upwards. The interpapillary processes were shallower and broader than natural, apparently the result of pressure from beneath. The great mass of this tumour appeared to consist of sarcomatous tissue, but an unusually distinct alveolation was present, the contained cells being epitheloid in character but not compressed or laminated, and without any resemblance to the superficial epithelium."

Dr. New MAN advised the use of thyrotomy in two stages, the first consisting of opening of the larynx, the second the removal of the growth after twenty-four or forty-eight hours, when a more thorough examination could be made. Endolaryngeal operations should hardly ever be employed for malignant disease, as the disease could not be completely removed. Thyrotomy might be successful in the early stages of cancer or sarcoma, but when the disease was advanced, Dr. Newman preferred partial or complete laryngectomy. In sarcoma, if the growth was still encapsuled, the operation of thyrotomy might be very successful.--Mr. LENNOX BROWNE congratulated the author on the success of his case, and said that his own experience of thyrectomy would make him hesitate to repeat the operation, for he believed that just as great a prolongation of life could be attained, with less discomfort, by a simple tracheotomy; but the operation of thyrotomy and removal of the malignant growth, the suggestion of which was due to Mr. Butlin, offered much greater hope of permanent relief, especially if performed sufficiently early; he meant so soon as positive microscopical evidence, or, failing that, laryngoscopic evidence and subjective symptoms gave clearindication of the nature of the disease.

\section{SECTION OF LARYNGOLOGY.}

Wednesday, Thursday and Friday, August 14th, $15 \mathrm{TH}$ AND 16TH.

Granules in the Pharynx.-Mr. L. A. LAwREnce read a paper based on 200 cases, mostly in hospital patients, which he had lately examined. No selection was made, the patients being for the most part warded for ordinary surgical and medical complaints. As regards the throat, it was to be presumed that the condition of these parts fairly well represented what might be noticed in the same classes in ordinary health. $\Lambda$ " granule" was defined as an elevation in the surface of the mucous membrane varying from the size of a small pin's head to that of a split pea or bean. Any hereditary diathesis, and notably struma, rheumatism, and gout, was said to predispose to the affection, the vagueness of the etiology being a proof of their ignorance on the subject. In the 200 cases examined, there were 168 examples of granular pharyngitis ; 30 patients were free from it; and in 2 young children the posterior wall of the pharynx could not be seen on account of the large size of the tonsils. Of the 168 , in 48 , or 28.57 per cent., there was personal or family history (sometimes both) of phthisis or struma ; in 34 , or 20.23 per cent., there was a history of struma or gout; in 20 there was a history of miscellaneous symptoms, or conditions such as cough, heart disease, asthma, and dropsy; and in the remaining 66 , or 39.28 per cent, there was a clean bill of health, both personal and hereditary. Of the 30 in whom no granules were seen, 9 , or 30 per cent., gave a history of phthisis or struma; 4 , or 13 per cent., of rheumatism; 3 gave other histories, and the remaining 14 were healthy. With respect to the alleged influence of phthisis, the healthy cases exhibited 30 per cent., the unhealthy 28.57 per cent. Phthisis might, therefore, be excluded as a factor. As regards rheumatism, the numbers were 13.33 per cent. of healthy, against 20.23 per cent. of unhealthy, showing an excess of the latter of only 7.9 per cent. The part played by rheumatism might, therefore, be practically ignored. Employment did not seem to have anything particular to do with the causation of granules. In those who used the voice to excess, though the granules might be a cause of alteration in the voice, it was not clear that the use of the voice was the cause of the granules. With regard to age, the numbers in each decade were $26,38,40,26,20,5,7$, with granules, as against 8,5 , $3,7,3,2,2$, without. Thus the earlier years up to 50 showed a marked preponderance, while after that age there was a sudden fall. The disease seemed to be common to all climates; this was certainly the case as regards Europe. Eighty-two men and 86 women out of 100 of each had the complaint, showing that the different occupations of the sexes-the exposure of men and the delicacy of women-had no influence for evil. In particular, tobacco as a factor was excluded by these figures. Diathesis, age, occupation, etc., having been excluded, the author suggested contagion as a possible cause. There was the analogy of granular lids, the granules being precisely similar in structure. In most cases the patient was not aware of the existence of the granules, but in 21 of the 200 cases symptoms were present, such as a feeling of dryness and stiffness in the throat, and occasionally a tickling sensation causing cough and even hoarseness. These might become aggravated so as to become seriously troublesome. The number of granules seemed to have some effect in the production of symptoms, as out of the 21 cases referred to, 13 had many and 5 had a few granules, whilst 3 had none. The granules might be situated in any part of the pharynx, might extend upwards to the naso-pharynx or down towards the osophagus; they might also be present in the palate and tonsils. The smaller varieties were by far the commonest, only 31 in 168 cases being of the larger sort. In 57 cases there were few-in some only one or twogranules, whilst double that number had many. Microscopically the granules showed simply lymphoid tissue. In the vast majority of cases no treatment was necessary. When symptoms were present they could be alleviated, but Mr. Lawrence did not think that a permanent cure could in the present state of knowledge be effected. Astringents were said to be useful, preference being given to chloride of zinc (gr. $\mathrm{xxx}$, ad. $\bar{\xi}$ ), applied as a paint, twice daily. "London paste" had also been used satisfactorily. An objection to the galvano-cautery was that hyperæmia at the injured site caused increased nutriticn in the neighbourhood, and then sometimes induced increased growth of the granules near. Mr. Butlin had lately used a form of Meyer's ring knife bent at a suitable angle for scraping the granules from the pharynx. The genera! health should be attended to in combination with thelocal treatment. -Dr. ScANes Sprcer said the statistical method adopted by $\mathrm{Mr}$. Lawrence was likely to lead to further knowledge of this affection, which was important, in that it caused considerable distress. Obstruction of the nose from any cause, whether structural and permanent or erectile and variable-perhaps only occurring at night in the recumbent position, and indicated by snoring, mouthbreathing, and dry mouth in the morning--was one of the most important factors in the production of granular pharyngitis ; and cure of the nasal obstruction was often followed by disappearance of the throat symptoms. In those cases in which obscure throat pain and discomfort existed, and there were none of the more commonly-recognised lesions of granular pharyngitis on the posterior wall, it was important to examine the base of the tongue where the "lingual tonsils" showed granular hypertrophic conditions similar to those seen in the pharyngeal adenoid masses, and destruction by the galvano-cautery was alike indicated in both cases if saline aperients and local application of iodine solutions did not soon remove the symptoms.-Dr. BARoN agreed with Dr. Spicer as to the importance of examining the back of the tongue in cases of granular pharyngitis. It frequently happened that 
patients submitted to a long and tedious course of treatment with no alleviation, and it was only when the outgrowths at the base of the tongue were systematically treated by astringents, caustics, or burning with red-hot wire, according to circumstances, that cure took place.-Dr. G. MACDONALD agreed with the remarks of the previous speakers as to the frequent association of lymphoid hypertrophy of the base of the tongue with granular pharyngitis; but dissented from Dr. Spicer's view as to nasal obstruction.

Throat and Nose Affections in Children in relation to Certain Derangements of Sleep, Temper, Spirits, Energy, Intellectual Power, and other Brain Functions.-Dr. SPICER, in reviewing the literature of the subject, proceeded to group the nervous derangements associated with throat and nose disorders in three classes: $(a)$ those indicative of irritation of nerve centres (disturbed sleep, night terrors, walking in sleep, excitability, stammering, etc.); (b) those indicative of exhaustion or debility of nerve centres (habitual headache, dizziness, depression, drowsiness, etc.); (c) those indicative of defective intellectual evolution and activity (slowness of, apprehension, weak memory, backwardness, etc.). The symptoms of irritation usually appeared first, and were followed by the others at varying intervals. Dr. Spicer was led to suspect an intimate connection between nervous derangements and affections of the naso-pharyngeal tracks by the frequency with which they coexisted, by the large proportion of idiot and weak-minded children in workhouses, etc., who possessed the characteristic physiognomy of adenoid vegetations, and by the frequency with which the symptoms referable to irritation disappeared, and the intellect, spirits, and temper improved, after cure of the throat or nose affection. Agnin, there was the analogy of the effects (such as languor, depression, etc.) produced by an ordinary cold on adults. Dr. Spicer had had many opportunities of observing that great mental depression accompanied long-standing interference with the patency of the nasal channels, and that this was removed on getting rid of the obstruction. Several cases from Dr. Spicer's own practice were cited in illustration of this; and instances were also given of slight forms of mental and nervous derangement occurring as complications of ordinary catarrh. Affections of the nose and throat were, therefore, of special importance during the growth of the organism. No definite statement could yet be made as to the proportion of cases in which such diseases were accompanied by nerve symptoms. Dr. Spicer did not think that the nose and throat affections and the nerve derangements were coincident results of constitutional weakness, but that the former were not only anterior in appearance to the latter, but exciting causes of them. He suggested that it might be possible that hypertrophic conditions in the naso-pharynx started abnormal stimuli, which passed along the terminal filaments of the trigeminus to the spheno-palatine ganglia, and were thence reflected along the vasomotor tracts, regulating the circulation of certain areas in the brain, so leading to abnormal alterations in the bloodsupply, with consequent changes in nutrition and derangements of function. Mr. Yearsley's suggestion that enlarged tonsils might cause pressure on the carotids, and thus diminish the supply of blood to the brain, was not improbable, and a similar effect might be produced by adenoid vegetations. The veins were still more likely to be pressed upon, and the venous blood dammed back to congest the nerve centres. The clearing up of nasal obstruction led to improvement of the general health, because the digestion was no longer disturbed by the swallowing of unhealthy secretions, and oxygenation of the blood was favoured by the inspired air being properly warmed and moistened. Dr. Spicer summed up his conclusion as follows: 1. That certain nose and throat affections in children were among the special causes of derangements of sleep, temper, spirits, energy, intellectual power, and other functions of the nervous system. 2. That long-standing derangement of these functions in the growing child implied a defect in the nutrition of the tissues, which would leave its mark on the nervous and mental faculties of the grown adult. 3. That if no sufficient cause of derangement of the above functions could be discovered elsewhere in the economy, the condition of the nose, naso-pharynx, and throat should not be dismissed without examination and consideration. 4. That the nasopharyngeal affections capable of causing the above derangements were principally chronic catarrh with erectile and hypertrophied condition of the mucous membrane, post-nasal adenoid vegetations, and enlarged tonsils. 5. That many of the above nervous derangements rapidly disappeared, and that others were ameliorated in a great number of cases by the cure of the local nose and throat disorder. 6. That the cases which were most likely to receive benefit from local treatment had generally also some of the following direct symptoms of nose and throat disorder: Perpetually open mouth and dropped jaw; habitual mouth breathing; snoring at night; dry mouth on waking in the morning; blockage of and inability to blow the nose; chronic and recurrent sore throat, colds, and coughs, and deafness-symptoms which demanded attention on their own account quite independently of any nerve derangement; further, the probability of benefit was increased if no sufficient cause of nerve irritation or exhaustion could be discovered elsewhere in the organism.-Dr. WARDEN stated that he quite concurred with Dr. Spicer's observations, especially with regard to disturbed and restless sleep, want of energy, extreme nervous irritability, and temper, the peculiar vacant look, open mouth, particularly in adenoid growths, and sometimes even in catarrhal affections and others. He related a case of post-nasal growth (muco-fibroma) of recurrent type, in which he had operated five or six times, and in which were strongly exemplified the prominent symptoms related by Dr. Scanes Spicer; the relief and almost total subsidence of the mental condition-fear and terrorso strongly existent in this case, being most marked after each operation, and returning gradually as the growths reappeared, vanishing again after another operation. The post-nasal growth was attached to the upper surface of the soft palate, and was suprapyramidal in shape, with a mucous polypus growing at the apex, and was also accompanied by considerable accumulation of nasal polypi.-Dr. Grevili.e MACDONALD, while congratulating Dr. Spicer on his clinical observations, and fully endorsing them, doubted whether they were yet justified in ascribing all the symptoms to reflex action. He quoted a case of epilepsy seen in consultation with Dr. John Davies, of Nottingham, and another of intellectual backwardness, both cured by removal of postnasal growths; yet he thought these and many others might be explained by the deficient oxygenation of the blood seen in most of such patients. He stated that many cases of snoring from nasal obstruction occurred during nasal respiration, the mouth, in spite of the stenosis of the nose, being tightly closed. In such cases the diminished barometric pressure within the air-chambers was evidenced by the recession of the anterior triangles during inspiration, this diminished air-supply in its turn leading to deficient aëration of the blood. He admitted, however, that many cases were explicable only on a theory of reflex action.

On Empyema of the Maxillary Sinus, with an Analysis of Fifteen Cases.-Dr. Greville MaCDONald began by asserting that the affection was to be considered a symptom rather than as a disease per se. His object was to bring forward certain symptoms that had hitherto been either misnamed or neglected. Among the former was the hypothetical "cleavage" of the middle turbinated bone, described as a consequence of "necrosing ethmoiditis"-so called-it really consisting in the development of an inflammatory neoplasm beneath the middle turbinated, pus from the antrum flowing from between the two structures. In cases where this was absent the pus might flow from above the middle turbinated. The principal subjective symptom was neuralgia and tenderness over the nasal or malar bones. He advocated opening the antrum from a bicuspid or molar socket, owing to difficulties in drainage when the pus was reached from the nose. He claimed a practical cure in ten of the fifteen cases.-Dr. ScANes SPICER said that unilateral purulent discharges from the nose were always suggestive of suppuration from the antrum. He did not think tenderness was common in suppuration of the antrum. The appearance of cleavage he believed to be due to inflammation and swelling of the bulla ethmoidalis.-Dr. W. RoBertson said Dr. Macdonald's paper reminded him of a case of antrum abscess which well illustrated the obscurity in whieh these cases were often involved. When the patient first came under notice she was suffering from well-marked asthmatic symptoms, together with loss of flesh. A delicacy of the lungs was spoken off by a well-known London consultant. On examining the nose a chronic blenorrhœa, complicated with polypi, was discovered. On removing the latter, the condition, although improved, was by no means cured-a most offensive discharge, with formation of crusts in left nostril, remaining. Recourse was had to syringing out the antrum, and, with the help of a little boracic lotion, about a tablespoonful and a half of very bad smelling pus was driven out of the left antrum. As strong exception was taken to the removal of a bicuspid, he perforated above this with a dentist's trephine, which gave good access to the cavity. For months, however, perfect irrigation with mild antiseptic had to be kept up, showing that lengthened treatment only would secure a successful result, even in 
perfectly benign cases, no matter where the situation of the artiticial aperture. Should the presence of pus during eating be offensive, the antrum could be opened under the inferior turbinated bone by means of an ordinary trocar bent to suit the direction of the cavity. There were no symptoms in the case beyond the offensive discharge from the nostril of same side, pointing to disease of the antrum.

New Olfactometer for Estimating Degree of Loss of Smell.Dr. Scanes SPICER exhibited to the Section, on behalf of Dr. ZWAARDEMAKER, of Utrecht, the olfactometer already described and figured in the Journal.

Rhinoscleroma. - Two cases of this rare affection were exhibited by Dr. Wr. Robertson (Newcastle): 1. The patient, M. L J., aged 33, presented total nasal obstruction, with deformity of nose; thickening and induration of alæ nasi, thickening and induration of walls of post-nasal space, which was thereby much reduced in size, with narrowing of Eustachian tubes; thickening with induration and cicatricial changes in larynx and trachea (raised semicircular band); tuberous swellings on upper gum. Symptoms unchanged for sixteen years. 2. The patient, M. H. J., aged 30 , deformity of nose, nasal obstruction on both sides. Approximation of velum palati (which showed cicatricial changes, and was drawn up), to post pharynx. Almost complete obliteration of post-nasal space. Roll-like contraction of epiglottis, cicatricial changes in larynx, semicircular raised band in trachea. Symptoms lasting thirteen years.-Mr. Lennox Browne said there were some signs which tended to a diagnosis of lupus, some to syphilis, and some to scrofula; and on casual examination he would have been inclined to say that the disorder was due to syphilis in a scrofulous subject. Nevertheless, Dr. Robertson had evidently paid such great attention to the subject, and had exhausted every means of arriving at a correct conclusion, that he (Mr. Browne) hesitated to offer a contrary opinion.Dr. SCANES SPICER said the loss of a considerable portion of the epiglottis, and the thickening of the stump in the elder patient, reminded him of cases of undoubted syphilis he had seen. It was an open question whether the application of nitrate of silver to the nares had not aided the cicatricial contraction which was apparent.-Dr. Robertson, in reply, said he was quite aware of the rarity of the disease, or at least of reported cases in this country. He should have been under the same disadvantage as many who had examined these cases had he not had the advantage lately of observing the ailment in Professor Schroetter's clinique in Vienna. That experience set at rest all doubt in his own mind as to these cases being veritable cases of rhinoscleroma. Mr. Lennox Browne had made a remark about venereal infection, but this had elicited only a history of gonorrhoa in the father at the age of 25 years, and even this must have become eradicated before his marriage, as witness the fact that his wife bore eight healthy children after marriage. Beyond that, syphilis as well as lupus, or a combination, as suggested by Mr. Lennox Browne, were both characterised by lesions leading to destruction of parts and loss of tissue. In these cases no loss of any part could be pointed out. The puckering of the soft palate was due to the cicatricial contraction on its upper surface, drawing it up and towards the posterior pharyngeal wall. Again, such hardness, with its characteristics, in the alæ nasi of the first patient, was entirely foreign to lesions due to either lupus or syphilis. The histological characters of the cells composing the infiltration differed from those of either syphilis or lupus, in that they were well formed and wanting in any indication of degeneration. It was not necessary to refer again to the duration of the process, the futility of treatment, and so on.

\section{THE ANALOGY OF SUMMER DIARRHGEA AND CHOLERA.}

By Sir WILLIAM MOORE, K.C.I.E., Q.H.P., Late Surgeon-General with the Government of Bombay.

Cholera is generally regarded as a disease characterised by certain definite symptoms, the principal distinctive features being cramps, suppression of urine, and evacuations resembling water in which rice has been boiled; and it has been customary to assert that a malady is not cholera when these so-called distinctive symptoms do not present, and also if the disease does not spread epidemically. From these conclusions I venture to dissent, and for the following reasons:

1. Many cases of cholera commence as diarrhoa, after which the so-called distinctive features of cholera develop; and the question, At what stage do sufferers from diarrhca become the victims of cholera? cannot be satisfactorily answered.

2. Terming a malady cholera only when certain symptoms occur is not consistent with what is known and admitted with regard to other diseases-diphtheria, scarlet fever, enteric fever, for examples-of all of which these are very mild cases, not presenting typical symptoms.

3. It must be recollected that cholera in its manifestations is a protean malady. Nothing better demonstrates this than the number of names under which it has been described, such as asphyxia, algide, bilious cholera, dry cholera, febris remittens choleroidea, hæmorrhagic cholera, white dysentery, etc.-terms all called into use by the varying nature of the malady. The disease appearing under different phases, and being described as it appeared, obtained a different appellation. The variability of cholera was noted by a Dr. Pennick, in 1832, on the presumed first appearance of the malady in this country. Pennick pointed out in what respect the English cholera differed from the previous Russian cholera. About the same time, Allan Webb asked in India: "Who can tell where cholera ends and plague begins, or distinguish between certain forms of cholera and fever?" Chevers states: "As the malarious poison produces all variations, from a fatal remittent to the mildest ague, so with the cholera poison." Sir Guyer Hunter writes: "As there are varying degrees of fever, so there are varying degrees of cholera. It may manifest itself by a slight looseness of the bowels, or as a sudden paralysis of the vasomotor system." In my Manual of the Diseases of India, I enumerate various peculiar phases-among which may be mentioned immediate death by collapse, without evacuations; cases resembling the sweating sickness of olden times; stools tinged with blood, or with bile; rice-water evacuations absent; cramps the only symptom; and, on the authority of Dickson, increased flow of urine.

4. The most recent surmise of the cause of cholera is Koch's comma microbe. And the previous surmise was Pettenkofer's theory, which postulates the earth with being the breeding place of the cholera germ. And now Pettenkofer has, it appears, adopted Koch's microbe as the germ. Pettenkofer's conditions are a certain degree of porosity of the ground, a certain amount of moisture indicated by a sinking of the ground water, and a certain amount of organic matter. Conditions which, by-the-bye, are not and cannot be fulfilled in various localities where cholera prevails. Now this is somewhat similar to the most recent theory of the causation of epidemic diarrhœa. Dr. Ballard, as the result of his recent investigations, says summer diarrhœa does not begin until a thermometer at 4 feet deep in the earth shows $56^{\circ}$, and that diarrhœe prevails so long as this earth temperature is maintained, no matter what the external temperature may be. Dr. Ballard also says the lighter the soil the more the diarrhœe (which is rather against the presumed salubrity of gravel soils). Lastly, Dr. Ballard expresses a provisional opinion that the essential cause of diarrhca resides in the upper layers of the earth, where it is intimately associated with the life process of some micro-organism not yet detected, captured, or isolated.

5 . Dr. Klein has investigated the pathology of summer diarrhoa, and he mentions empty intestines, or containing only fluid, follicular ulcerations, congested spots, denudation of epithelium, and degenerative kidney changes. Such conditions are mostly applicable to cholera.

6. Although the diagnosis of a typical case of cholera from a typical case of diarrhœa is sufficiently easy, in seasons of epidemic no one can say whether or not the latter will become the former. Moreover, there are eases which so resemble cholera that they would be "unhesitatingly recorded as cholera if cholera were epidemic at the time." This remark actually occurs in Reynolds's System of Medicine. Ziemssen observes: "It is impossible to distinguish one from the other, unless regard be had to the absence or presence of cholera." Sir Joseph Fayrer says "the conditions are very similar if not actually identical." Flint observes: "It is by no means easy to distinguish the first case of cholera from cholera nostras.

7. If recovery from bad diarrhœa takes place a low febrile condition often ensues, just as results after Asiatic cholera.

8. The best treatment for summer diarrhœa, whether hygienic or medicinal, is that found most suitable in cholera. 